\title{
Affinity Constants of Naturally Acquired and Vaccine-Induced Anti- Pseudomonas aeruginosa Antibodies in Healthy Adults and Cystic Fibrosis Patients
}

\author{
Urs Bruderer, Stanley J. Cryz, Jr., Urs B. Schaad, \\ Markus Deusinger, John U. Que, and Alois B. Lang
}

Swiss Serum and Vaccine Institute and Department of Pediatrics. Inselspital, University of Berne. Switzerland

Naturally acquired anti-Pseudomonas aeruginosa antibody fails to afford protection against repeated $P$. aeruginosa bronchopulmonary exacerbations in cystic fibrosis (CF) patients. In an effort to explain this phenomenon, the titer and affinity constants of serum anti-lipopolysaccharide (LPS) IgG were determined in five study groups: healthy adults before and after immunization with a polyvalent LPS-based vaccine, healthy noncolonized CF patients before and after immunization, nonimmunized CF patients with significantly elevated anti-LPS antibody titers without documented colonization, recently colonized CF patients before and after immunization, and nonimmunized $\mathrm{CF}$ patients chronically colonized with $P$. aeruginosa. Immunization elicited a significant rise in total anti-LPS immunoglobulin levels and affinity constants in both healthy adults and CF patients. Although chronically colonized patients had elevated levels of total anti-LPS antibody, these antibodies possessed affinities at least 100-fold less than those of vaccine-induced antibodies.

Bronchopulmonary infections due to Pseudomonas aeruginosa are a leading cause of morbidity and mortality among cystic fibrosis (CF) patients [1]. Overt disease caused by rough mucoid strains of $P$. aeruginosa is preceded by colonization of the upper respiratory tract with smooth strains [2]. Colonization and pulmonary exacerbations stimulate a rise in serum antibody levels to a number of somatic and extracellular antigens including lipopolysaccharide (LPS), alginate, and toxin $\mathrm{A}[3,4]$. Such antibodies do not appear to prevent or ameliorate subsequent infections.

Several laboratories have found a positive correlation between severe disease and elevated anti- $P$. aeruginosa antibody levels $[5,6]$. In addition, circulating immune complexes formed during pulmonary exacerbations may contribute substantially to organ damage $[6,7]$. The above has led to the belief that such antibodies are detrimental to the host, although the precise reason such antibodies are not protective is not known. The most widely held belief is that most naturally acquired antibodies possess a greatly reduced opsonic capacity $[8,9]$. However, serum from colonized CF patients can protect mice against experimental $P$. aeruginosa when passively transferred [5]. Furthermore, recent studies have found that $\mathrm{CF}$ serum can support the uptake of $P$. aeru-

Received II October 199I; revised 26 February 1992.

The study was approved by the ethical review committees of the Inselspital and Tiefenau Hospital (Berne). Written informed consent was obtained from all subjects or their parents.

Reprints or correspondence: Dr. Urs Bruderer, Department of Immunology. Swiss Serum and Vaccine Institute. P.O. Box 2707. CH-3001 Berne. Switzerland.

The Journal of Infectious Diseases 1992; 166:344-9 (C) 1992 by The University of Chicago. All rights reserved. $0022-1899 / 92 / 6602-0017 \$ 01.00$ ginosa by polymorphonuclear leukocytes from healthy adults [8]. Therefore, at least a subpopulation of naturally acquired antibodies possess functionally relevant traits.

A number of approaches, including immunization, are being evaluated in an effort to control recurrent $P$. aeruginosa infections in CF patients [10-12]. Of critical importance is to establish the long-term safety of such vaccines and to demonstrate that immunization engenders antibodies with desired functional attributes. We have recently described the safety and immunogenicity of an octavalent $\mathrm{O}$-polysaccharidetoxin A conjugate vaccine in young noncolonized $\mathrm{CF}$ patients [13]. In the present study we compared the affinity constants of naturally acquired and vaccine-induced antiLPS antibodies.

\section{Materials and Methods}

Vaccine. The synthesis, safety, and immunogenicity of the polyvalent $P$. aeruginosa $O$-polysaccharide-toxin A conjugate vaccine have been described previously $[14,15]$. Each dose contained $25 \mu \mathrm{g}$ of O-polysaccharide of the following international antigen typing serotypes (IATS): 1, 2, 3, 4, 6, 7, 10, and 11 .

LPS. LPS from P. aeruginosa PA220 (IATS 6) and P. aeruginosa 27313 (IATS 11) was isolated as described [16]. The LPS preparations contained $<2 \%(\mathrm{wt} / \mathrm{wt})$ protein and nucleic acid.

Determination of anti-LPS antibody titers. Serum antibodies specific for LPS were quantitated by ELISA as described elsewhere $[5,17,18]$. For coating, purified LPS $(5 \mathrm{mg} / \mathrm{ml})$ dissolved in $36 \mathrm{~m} M$ triethylamine was used. Microtiter plates (Immulon; Dynatech, Buchs, Switzerland) were used for incubation: each well contained $100 \mu \mathrm{l}$ of a solution of $1 \mu \mathrm{g}$ of LPS $/ \mathrm{ml}$ of $0.1 M$ sodium carbonate buffer, $\mathrm{pH}$ 9.6.

Determination of antibody affinities. Apparent affinity constants were obtained by ELISA-based determinations of the re- 
ciprocal of the molar antigen concentration resulting in $50 \%$ inhibition of antibody binding. Serum samples were titrated in PA220 LPS-coated microtiter plates as described above, and dilutions resulting in an OD of $0.8-1.2$ were used. The percentage of binding in the presence of LPS was determined by extrapolation to a standard curve constructed from parallel experiments with known percentages of the concentration of antibodies used in the inhibition experiments [19]. The concentration of LPS resulting in 50\% inhibition of the antibody binding was determined as described by Reed and Muench [20]. The affinity constants of monoclonal antibodies determined by this method have been shown to be in close agreement with the intrinsic affinity constant measured by Farr assay and fluorescence quenching assays [2 l]. Calculations of the molar concentrations were based on the assumed molecular weight of 10,000 for LPS as described by Schwartzer et al. [22].

The range of detectable affinities in polyclonal sera depends on the concentration of the antigen in the solid phase [23]. A concentration of PA220 LPS $(1 \mu \mathrm{g} / \mathrm{ml})$ was chosen, which allowed the detection of antibodies over a range of $10^{4}$ to $>10^{8} M^{-1}$.

The specificity of this assay system was verified with monoclonal antibodies specific for $P$. aeruginosa LPS [17, 18]. Only antibodies specific for IATS 6 LPS or those that recognize antigenic determinants common to most serotypes of $P$. aeruginosa LPS gave a signal in this assay system.

Patients and clinical studies. CF patients were enrolled from those attending the CF clinic at the Inselspital. Healthy adults were staff members at the Tiefenau Hospital, Berne. Radiologic and pulmonary scores (Crispin-Norman and Bernese) and colonization with $P$. aeruginosa were determined at the time of immunization and at yearly intervals thereafter as previously described [5]. A patient classified as colonized had $P$. aeruginosa isolated from sputum or saliva samples on multiple occasions for at least 5 years before entry into this study. A patient was classified as noncolonized when three conditions were met: no history of $P$. aeruginosa isolated from either sputum or saliva samples, no overt bronchopulmonary infection attributable to $P$. aerugin$o s a$, and possession of low IgG antibody levels to nine serotypes of $P$. aeruginosa LPS frequently associated with infections in $\mathrm{CF}$ patients and to exotoxin $A$. The mean ages (ranges) for the patient groups were as follows: noncolonized immunized, 6.3 years (2-11); colonized nonimmunized, 13.2 (7-22); "questionably" colonized (significantly elevated anti-LPS antibody titers without documented colonization), 18.5 (6.5-30); colonized immunized, 8.5 (7-9).

Healthy subjects received a vaccine dose equal to $25 \mu \mathrm{g}$ of $\mathrm{O}-$ polysaccharide per vaccine serotype ( $200 \mu \mathrm{g}$ total) on days 0 and 28 . CF patients received $12.5 \mu \mathrm{g}$ of $\mathrm{O}$-polysaccharide per vaccine serotype on days 0 and 56 . The vaccine was administered intramuscularly in the deltoid area in $0.5 \mathrm{ml}$ of vehicle. Serum samples were collected 2-4 weeks after each immunization. Clinical scores for CF patients were obtained at the time of primary immunization.

Opsonophagocytic assay. The ability of sera from CF patients to support the uptake and killing of $P$. aeruginosa PA220 (IATS 6) by polymorphonuclear leukocytes from healthy adults was measured as described elsewhere [10] with the following modifications. The final concentration of normal human serum was $1 \%$ and the sampling times were at 0 and $90 \mathrm{~min}$. Test sera were evaluated at the following dilutions: $1: 12,1: 36,1: 108$, $1: 324$, and $1: 972$. Opsonic titer was defined as the reciprocal of the highest dilution of serum that resulted in $\geqslant 70 \%$ killing of the initial bacterial inoculum.

Statistical analysis. Significance between IgG ELISA titers and affinity constants before and after immunization was determined by the paired $t$ test. Significance between immunized and nonimmunized groups was determined by the single-tailed $t$ test for groups.

\section{Results}

Characteristics of the five study groups are shown in table 1. The IATS 6 LPS was selected as representative antigen for the following reasons: it is the predominating serotype of $P$. aeruginosa associated with pulmonary infections at the Inselspital CF Clinic, all colonized CF patients had elevated antiIATS 6 LPS antibody, and the IATS 6 vaccine component was found to be highly immunogenic in healthy adults and CF patients. Healthy adults had low levels (mean titer, 67) of naturally acquired anti-LPS antibodies. Noncolonized CF patients, whose mean antibody titer was comparable to that of healthy adults, had clinical scores within the normal range. A group of 6 CF patients was included in this study whose colonization statuses were unclear (questionably colonized). Although $P$. aeruginosa was never isolated, all had elevated IgG antibody titers to a bank of nine $P$. aeruginosa LPS antigens including IATS 6 (mean titer, 970), strongly suggestive of prior subclinical exposure or current low-level colonization. In addition, their baseline clinical scores fell between those of noncolonized and colonized CF patients. Also included were 2 patients recently colonized with IATS $6 P$. aeruginosa (4-5 months before immunization). The final group was 8 patients colonized for a mean of 6.4 years. This group had markedly reduced clinical scores and elevated anti-IATS 6 LPS IgG antibody titers.

Local reactions, characterized by pain and swelling at the injection site, were classified as mild to moderate and were associated with roughly half of the vaccinations. Reactions manifested by the 2 colonized patients who were immunized were similar in severity and duration to those observed for the noncolonized patients. No systemic reactions were reported.

The anti-IATS 6 immune response to immunization with the $P$. aeruginosa polyvalent vaccine and affinity constants for baseline and postimmunization serum samples are shown in table 2 . Immunization of healthy adults or noncolonized CF patients resulted in a significant $(P<.05)$ rise in $\operatorname{IgG}$ antibody levels. There was no difference in the magnitude of the immune response between these two groups $(P>.05)$. The 2 newly colonized CF patients (whose baseline titers were similar to those of the above groups) also responded 
Table 1. Clinical characteristics of study groups.

\begin{tabular}{|c|c|c|c|}
\hline \multirow[b]{2}{*}{ Group $(n)$} & \multicolumn{2}{|c|}{ Mean base clinical score (range) } & \multirow{2}{*}{$\begin{array}{c}\text { Mean baseline IgG } \\
\text { titer (range) }\end{array}$} \\
\hline & Bernese & Crispin-Norman & \\
\hline Healthy adults, noncolonized, immunized ( 6 ) & ND & ND & $67(<40-350)$ \\
\hline \multicolumn{4}{|l|}{ CF patients } \\
\hline Noncolonized. immunized (7) & $23.1(22-24)$ & $4.8(3-8)$ & $31(<40-130)$ \\
\hline Questionably colonized, nonimmunized (6) & $20.7(16-24)$ & $8.2(4-13)$ & $970(146-7166)$ \\
\hline Colonized 0.4 years, immunized (2) & $23.5(23,24)$ & 4.0 & 60 \\
\hline Colonized 6.4 years, nonimmunized ( 8 ) & $14.3(11-20)$ & $15.9(8-21)$ & $2108(1086-10.347)$ \\
\hline
\end{tabular}

NOTE. Baseline titers are at time of enrollment into study for those nonimmunized or at time of immunization, ND = not determined. Questionably colonized: significantly elevated antibody titers without documented colonization.

with a vigorous antibody response to vaccination. Subjects in all three groups had extremely low baseline affinity constants $\left(\leqslant 0.02 \times 10^{6} \mathrm{M}^{-1}\right)$. The mean affinity constant for normal adults and noncolonized CF patients increased $>10,000-$ fold after immunization $(P<.001)$. Both newly colonized patients responded with a pronounced rise in affinity after immunization.

Chronically colonized and questionably colonized CF patients had baseline anti-IATS 6 antibody titers significantly higher $(P<.05)$ than those of healthy adults or noncolonized patients. Furthermore, their baseline titers exceeded those attained by healthy adults and noncolonized patients after immunization. Baseline affinity constants for this chronically colonized group were significantly higher $(P$ $=.027$ ) than baseline levels for either healthy adults or noncolonized patients, while a similar comparison for the questionably colonized group approached significance $(P$ $=.077$ ).

Immunized healthy adults or noncolonized CF patients possessed mean affinity constants at least 35 -fold higher than those seen for colonized patients $(P<.001)$. Both recently colonized CF patients also showed a marked increase (>1000-fold) in antibody affinity after immunization. A similar trend was seen when IATS 11 LPS was used as a test antigen (data not shown).

It was of interest to determine if the induction of high-affinity antibody by vaccination was associated with an increase in functional capacity. Therefore, sera from noncolonized immunized patients and colonized nonimmunized patients with high levels of anti-IATS $6 \mathrm{IgG}$ antibody were tested for their ability to promote the opsonophagocytic killing of an IATS 6 strain of $P$. aeruginosa (table 3). The sera from only 2 of 8 colonized patients were able to promote the uptake and killing of the test strain, whereas sera from all 3 immunized patients expressed opsonic activity. The ratio of antibody to opsonic titer for immunized patients and for colonized nonimmunized patients ranged from 12 to 18 and from 55 to $>446$, respectively. These data indicate that the high-affinity antibody induced by vaccination possesses the desired functional attributes.

The kinetics of the immune response induced by vaccination pertaining to both $\operatorname{IgG}$ antibody titer and mean affinity constant was studied. Sequential serum samples were available for I patient who became colonized with an IATS $6 P$.

Table 2. Anti-P. aeruginosa (international antigen typing serotype 6) IgG antibody response and affinity after vaccination and induced naturally.

\begin{tabular}{|c|c|c|c|c|}
\hline \multirow[b]{2}{*}{ Group } & \multicolumn{2}{|c|}{ Geometric mean IgG titer (range) } & \multicolumn{2}{|c|}{ Mean affinity constant (range) } \\
\hline & Baseline & $\begin{array}{l}\text { After second } \\
\text { immunization }\end{array}$ & Baseline & $\begin{array}{l}\text { After second } \\
\text { immunization }\end{array}$ \\
\hline Healthy adults & $67(<40-350)$ & $561(78-1500)$ & $0.02(<0.01-0.01)$ & $238(50-650)$ \\
\hline \multicolumn{5}{|l|}{ CF patients } \\
\hline Noncolonized & $31(<40-130)$ & $432(100-2060)$ & $<0.01$ & $332(110-640)$ \\
\hline Newly colonized & 60 & $2465(1280,3650)$ & $<0.01$ & $319(220,417)$ \\
\hline Questionably colonized & $970(146-7166)$ & NA & $1.1(<0.01-18.0)$ & NA \\
\hline Chronically colonized & $2108(1086-10,347)$ & NA & $6.3(1.1-49.0)$ & NA \\
\hline
\end{tabular}

NOTE. Affinity constants are $\times 10^{6} \mathrm{M}^{-1}$. NA $=$ not applicable (groups were not immunized). Questionably colonized: significantly elevated antibody titers without documented colonization. 
Table 3. Opsonophagocytic killing of $P$. aeruginosa (international antigen typing serotype 6 ) mediated by serum from immunized noncolonized and nonimmunized colonized CF patients.

\begin{tabular}{lccc}
\hline Group, subject & $\begin{array}{c}\text { Ratio } \\
\text { Anti-LPS IgG } \\
\text { antibody titer* }\end{array}$ & $\begin{array}{c}\text { Opsonic } \\
\text { titer }^{\dagger}\end{array}$ & $\begin{array}{c}\text { Ratibody titer/ } \\
\text { opsonic titer }^{\dagger}\end{array}$ \\
\hline Colonized nonimmunized & & & \\
1 & 10.347 & 36 & 287 \\
2 & 1086 & $<12$ & $>91$ \\
3 & 4302 & $<12$ & $>359$ \\
4 & 2518 & $<12$ & $>210$ \\
5 & 2622 & $<12$ & $>222$ \\
6 & 2723 & $<12$ & $>227$ \\
7 & 5347 & $<12$ & $>446$ \\
8 & 1972 & 36 & 55 \\
Noncolonized immunized & & & 17 \\
1 & 200 & 12 & 12 \\
2 & 1300 & 12 & 18 \\
3 & 210 & 108 & \\
\hline
\end{tabular}

* Samples from immunized patients were obtained $\sim 1$ month after second immunization (average of three independent determinations). LPS = lipopolysaccharide.

${ }^{\dagger}$ Expressed as reciprocal of highest dilution of serum resulting in $\geq 70 \%$ killing.

${ }^{\ddagger}$ Derived by dividing antibody titer by opsonic titer.

aeruginos $a$ and elected to be vaccinated $\sim 5$ months later (figure 1). Anti-LPS IgG antibody titer did not increase during the first 5 months of colonization, although nonmucoid $P$. aeruginosa was repeatedly obtained from sputum cultures during this time. Before immunization, this patient's affinity constant remained at $<0.1 \times 10^{6} \mathrm{M}^{-1}$. Immunization evoked a parallel rise in IgG ELISA titer and affinity constant, although the latter was far more pronounced. After primary immunization, the IgG ELISA titer increased from 110 to 280 , while affinity rose from $<0.1$ to $21 \times 10^{6} \mathrm{M}^{-1}$. A booster dose of vaccine resulted in a further substantial rise in both parameters.

The 7 immunized noncolonized patients have been followed for 25-28 months after vaccination. All remain noncolonized, and there was no significant change in their clinical scores. Similarly, no change in clinical status was observed for the 2 colonized patients who were immunized.

\section{Discussion}

Patients with CF have an opsonic defect specific for $P$. aeruginosa that extends to blood-derived polymorphonuclear leukocytes and lung macrophages $[24,25]$. This deficiency is at least partly responsible for the observation that CF patients experience repeated pulmonary exacerbations even when possessing high levels of anti- $P$. aeruginosa antibodies.

Naturally acquired anti-LPS and anti-alginate antibodies in CF patients consist of a heterogeneous population of op- sonic and nonopsonic antibodies, with the latter predominating in most individuals $[8,26]$. Nonopsonic antibodies are believed to contribute substantially to bronchopulmonary disease in CF by recruitment of inflammatory cells or by immune complex formation [7, 8, 27, 28]. However, some CF patients can mount what appears to be a protective opsonic antibody response [26]. If this is indeed the case, immunologic intervention in the form of immunization may be of benefit provided that antibodies expressing the desired functional attributes can be engendered.

Several possible mechanisms have been proposed to explain the CF opsonic defect. Fick et al. [29] have proposed that opsonization is compromised by a weak interaction between antibody and $\mathrm{Fc}$ receptors expressed by phagocytic cells. Moss and coworkers [30, 31] have noted an altered antibody isotype distribution in CF patients. Certain isotypes of antibody, specifically IgG2, were found to be far more efficient at opsonizing both mucoid and nonmucoid $P$. aeruginosa [8].

The functional traits expressed by a given antibody will be determined not only by its epitope specificity and isotype but also by its affinity [32]. High-affinity antibodies are far more effective than low-affinity antibodies at binding complement [33]. This may be of considerable significance since opsonophagocytic killing of mucoid and nonmucoid $P$. aeruginosa is a complement-dependent process $[34,35]$. Previous data suggesting that anti- $P$. aeruginosa antibodies from $C F$ patients may not interact properly with $\mathrm{Fc}$ receptors on phagocytic cells [29] prompted us to investigate the affinity of anti-

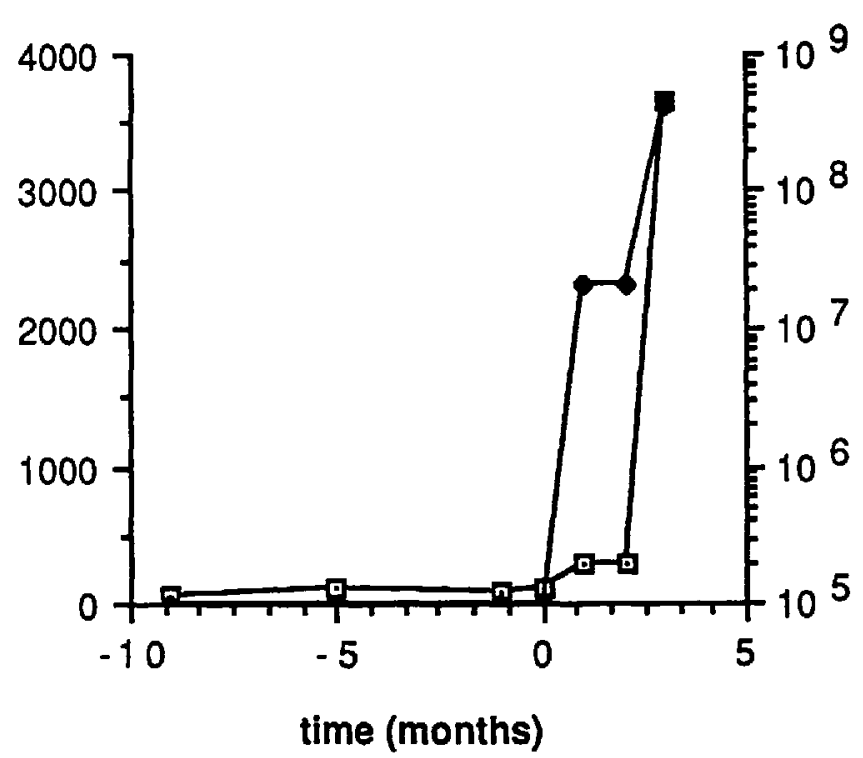

Figure 1. Antibody titers $(\square)$ and affinities $(\bullet)$ of an immunized CF patient with previous infection. International antigen typing serotype $6 P$. aeruginosa was detected 5 months $(-5)$ before primary immunization ( 0 ). A secondary vaccination was administered 2 months later. 
LPS antibodies. The fact that we had access to serum derived from CF patients immunized with an LPS-based conjugate vaccine gave us the unique opportunity to compare the affinities of naturally acquired and vaccine-induced antibodies.

Healthy adults and noncolonized CF patients possessed low baseline levels of anti-IATS 6 antibodies with low mean affinity constants. Immunization engendered an 8- to 14fold rise in IgG titer but a 10,000-fold rise in mean affinity. The immune response in both groups was comparable, indicating that noncolonized $C F$ patients can respond adequately to a parenterally administered $P$. aeruginosa LPSbased vaccine. Two patients recently colonized with IATS 6 $P$. aeruginosa also responded to vaccination with high-affinity antibodies. Even though postimmunization serum from healthy adults or CF patients contained somewhat lower total IgG levels than seen in serum from nonvaccinated colonized CF patients, mean affinity constants for the former groups were significantly higher. The ratio of $\mathrm{IgG}$ titer to mean affinity constant was 334:1 for colonized patients and 2.3:1 and 1.3:1 for postimmunization sera from healthy adults and noncolonized patients, respectively. Therefore, it appears that a far greater proportion of vaccine-induced antibodies than of those acquired after colonization or infection are of high affinity.

Such high-affinity antibodies appear to mediate the opsonophagocytic killing of $P$. aeruginosa. The sera from all $3 \mathrm{im}-$ munized noncolonized patients supported the uptake and killing of an IATS 6 test strain, whereas the sera from only 2 of the 8 nonimmunized chronically colonized patients did so. Numerous prior studies have documented a defect in the ability of CF sera to promote opsonophagocytic killing of $P$. aeruginosa $[8,9,24]$. Although the nature of this defect is not precisely known, it can apparently be overcome by inducing antibody through vaccination.

CF patients appear to have no innate defect in their ability to produce high-affinity anti-LPS antibodies. This would suggest that the route by which the immune system is stimulated is of critical importance. Repeated persistent exposure to $P$. aeruginosa in the respiratory tract does not appear to routinely engender high-affinity antibodies. It is important to note that 2 of 8 chronically colonized patients did possess an intermediate mean affinity constant $\left(25-50 \times 10^{6} \mathrm{M}^{-1}\right)$. This may indicate a slow maturation of high-affinity antibodies in this patient population, since these patients had been colonized the longest. Thus, one may speculate that the early development of opsonic high-affinity antibodies leads to protection against $P$. aeruginosa infection among a subgroup of $\mathrm{CF}$ patients [26]. However, most CF patients produce lowaffinity nonopsonic antibodies after early exposure to $P$. aeruginosa, which would not prevent colonization or subsequent infection.

The above findings may have an important bearing on the potential of active immunization to prevent $P$. aeruginosa infections in CF patients. The O-polysaccharide-toxin A vaccine was capable of stimulating a rapid rise in high-affinity and opsonic antibodies in young noncolonized CF patients. Booster doses of vaccine resulted in a rise in both total anti-LPS IgG antibody and mean affinity constant without a detrimental effect on clinical status. Vaccination induced primarily IgGI and IgG2 anti-LPS antibodies (unpublished data), which are capable of supporting opsonophagocytosis of $P$. aeruginosa. Use of such a vaccine, which engenders functional antibody, may circumvent potential hazards associated with the immunization of $\mathrm{CF}$ patients $[6,7,11]$.

\section{References}

1. Marks MI. The pathogenesis and treatment of pulmonary infections in patients with cystic fibrosis. J Pediatr 1981;98:173-9.

2. Pier GB, Des Jardins D, Aquilar T, Barnard M, Speert DP. Polysaccharide surface antigens expressed by nonmucoid isolates of $P_{\text {seudo- }}$ monas aeruginosa from cystic fibrosis patients. J Clin Microbiol 1986;24: 189-96.

3. Pedersen SS, Espersen F, Høiby N, Jensen T. Immunoglobulin A and immunoglobulin $\mathrm{G}$ antibody response to alginates from Pseudomonas aeruginosa in patients with cystic fibrosis. J Clin Microbiol 1990;28:747-55.

4. Fomsgaard A, Høiby N, Shand GH, Conrad RS, Galanos C. Longitudinal study of antibody response to lipopolysaccharides during chronic Pseudomonas aeruginosa lung infection in cystic fibrosis. Infect Immun 1988;56:2270-8.

5. Schaad UB. Lang AB, Wedgwood J, Buehlmann U, Fürer E. Serotypespecific serum IgG antibodies to lipopolysaccharides of Psetudomonas aeruginosa in cystic fibrosis: correlation to disease, subclass distribution, and experimental protective capacity. Pediatr Res 1990;27: 508-13.

6. van Bever HP. Gigase PL. DeClerck LS, Bridts CH, Franckx H, Stevens WJ. Immune complexes and Pseudomonas aeruginosa antibodies in cystic fibrosis. Arch Dis Child 1988;63:1222-8.

7. Høiby N, Döring G, Schiotz PO. The role of immune complexes in the pathogenesis of bacterial infections. Annu Rev Microbiol 1986:40:29-53.

8. Eichler I, Joris L, Hsu YP, van Wye J. Bran R, Moss R. Nonopsonic antibodies in cystic fibrosis. Pseudomonas aeruginosa lipopolysaccharide-specific immunoglobulin $G$ antibodies from infected patient sera inhibit neutrophil oxidative response. J Clin Invest 1989;84:1794-804.

9. Fick RB, Naegel GP. Squire SU, Wood RE, Gee JBL, Reynulds HY. Proteins of cystic fibrosis respiratory tract. Fragmented immunoglobulin $\mathrm{G}$ opsonic antibody causing defective opsonophagocytosis. J Clin Invest 1984;74:236-48.

10. Cryz SJ Jr, Fürer E, Que JU. Synthesis and characterization of a Pseudomonas aeruginosa alginate-toxin A conjugate vaccine. Infect Immun 1991;59:45-50.

11. Langford DT, Hiller J. Prospective, controlled study of a polyvalent pseudomonas vaccine in cystic fibrosis-three year results. Arch Dis Child 1989;59:1131-4.

12. Pier GB. Small GJ, Warren HB. Protection against mucoid Psetudo monas aeruginosa in rodent models of endobronchial infection. Science 1990;249:537-40.

13. Schaad UB, Lang AB, Wedgwood J, et al. Safety and immunogenicity of Pseudomonas aeruginosa conjugate A vaccine in cystic fibrosis. Lancet 1991;338:1236-7.

14. Gottlieb DJ. Cryz SJ Jr. Fürer E. et al. Immunity against $P_{\text {seudomonas }}$ aeruginosa adoptively transferred to bone marrow transplant recipients. Blood 1990;76:2470-5. 
15. Cryz SJ Jr, Sadoff JC, Cross AS, Fürer E. Safety and immunogenicity of a polyvalent Pseudomonas aeruginosa O-polysaccharide-toxin $\mathrm{A}$ vaccine in humans. Antibiot Chemother 1989;42:177-83.

16. Cryz SJ Jr, Lang AB, Sadoff JC, Germanier R, Fürer E. Vaccine potential of Pseudomonas aeruginosa O-polysaccharide-toxin A conjugates. Infect Immun 1987;55: 1547-51.

17. Lang AB, Fürer E, Senyk G, Larrick JW, Cryz SJ Jr. Systematic generation of antigen specific human monoclonal antibodies with therapeutic activities using active immunization. Hum Antibodies Hybridomas 1990;1:96-103.

18. Lang AB, Bruderer U. Fürer E. Larrick JW. Cryz SJ Jr. Immunoprotective capacities of human and murine monoclonal antibodies recognizing serotype specific and common determinants of gram-negative bacteria. In: Borrebaeck C. Larrick JW. eds. Therapeutic monoclonal antibodies. New York: Stockton Press, 1990:223-34.

19. Bruderer U. Fürer E. Cryz SJ Jr, Lang AB. Qualitative analysis of antibody binding. An in vitro assay for the evaluation and development of vaccines. J Immunol Methods 1990;133:263-8.

20. Reed LJ, Muench HA. A simple method of estimating fifty per cent endpoints. Am J Hyg 1938;27:493-7.

21. Nieto A, Gaya A, Jansa M, Moreno C, Vives J. Direct measurement of antibody affinity distribution by hapten-inhibition enzyme immunoassay. Mol Immunol 1984;21:537-43.

22. Schwartzer TA, Alcid DV. Numsuwan V, Glocke DJ. Immunochemical specificity of human antibodies to lipopolysaccharide from the J5 rough mutant of Escherichia coli 0 I I I:B4. J Infect Dis 1989;159:3542.

23. Kim YT, Weberlin TP, Siskind GW. Distribution of affinities. II. Fractionation of antibody with respect to its hapten binding affinity. Immunochemistry 1974;11:685-90.

24. Thomassen MJ, Boxerbaum B, Denko CA, Kuchenbrod PJ, Dearborn DG, Wood RE. Inhibitory effect of cystic fibrosis serum on Pseudomonas phagocytosis by rabbit and human alveolar macrophages. Pediatr Res 1979; 1 3:1085-8.

25. Cassino RJJ, Sordelli DO. Macri CN. Kohan M, Dillon MH, Pivetta
OH. Pulmonary nonspecific defense mechanisms in cystic fibrosis. I. Phagocytic capacity of alveolar macrophages and neutrophils. Pediatr Res 1980;14:1212-5.

26. Pier GB, Saunders JM, Ames P, et al. Opsonophagocytic killing antibody to Pseudomonas aeruginosa mucoid exopolysaccharide in older noncolonized patients with cystic fibrosis. $N$ Engl J Med 1987;317:793-8.

27. Thomassen MJ, Denko CA, Wood RE, Sherman JA. Phagocytosis of Pseudomonas aeruginosa by polymorphonuclear leukocytes and monocytes: effect of cystic fibrosis serum. Infect Immun 1982;38:802-5.

28. Moss RB, Lewiston NJ. Immunopathology of cystic fibrosis. In: Shapiro E, Wilson GB, eds. Immunological aspects of cystic fibrosis. Boca Raton, FL: CRC Press, 1984:5-27.

29. Fick RB, Naegel GP, Matthay RA, Reynolds HY. Cystic fibrosis Pseudomonas opsonins. Inhibitory nature in an in vitro phagocytic assay. J Clin Invest 1981;68:899-914.

30. Moss RB, Hsu YP, Van Eede PH, Van Leeuwen MV, Lewiston NJ, De Lange $\mathbf{G}$. Altered antibody isotype in cystic fibrosis: impaired natural antibody response to polysaccharide antigens. Pediatr Res 1987:22:708-13.

31. Moss RB, Hsu YP, Sullivan MM, Lewiston NJ. Altered antibody isotype in cystic fibrosis: possible role in opsonic deficiency. Pediatr Res 1986;20:453-9.

32. Steward MW, Steensgaard J. Antibody affinity: thermodynamic aspects and biological significance. Boca Raton, FL: CRC Press, 1983.

33. Fauci AS, Frank MM, Johnson JS. The relationship between antibody affinity and efficiency of complement fixation. $J$ Immunol 1970;105:2 15-22.

34. Young LS, Armstrong D. Human immunity to Pseudomonas aeruginosa. I. In vitro interaction of bactcria, polymorphonuclear leukocytes and serum factors. J Infect Dis 1972;126:257-76.

35. Ames P, Des Jardins D, Pier GB. Opsonophagocytic killing activity of rabbit antibody to Pseudomonas aenginosa mucoid exopolysaccharide. Infect Immun 1985:49:281-5. 